\title{
Lectotipificación de los nombres descritos por J. Barbosa Rodrigues en la obra "Palmae novae Paraguayenses"
}

\author{
Fred Stauffer, Lorenzo Ramella, Evandro Ferreira \& Larry Noblick
}

\begin{abstract}
STAUFFER, F., L. RAMELLA, E. FERREIRA \& L. NOBLICK (2016). Lectotypification of names proposed by J. Barbosa Rodrigues in the publication "Palmae novae Paraguayenses". Candollea 71: 105-115. In Spanish, English and Spanish abstracts. DOI: http://dx.doi.org/10.15553/c2016v711a13

In the frame of the treatment of the family Palmae to be published in the Flora del Paraguay, lectotypes are designated for the following names described by J. Barbosa Rodrigues in the publication "Palmae novae Paraguayenses": Attalea guaranitica Barb. Rodr., Bactris anisitsii Barb. Rodr., Cocos paraguayensis Barb. Rodr. and Cocos sapida Barb. Rodr. The latter is here proposed as a new synonym of the widely distributed South-eastern Brazil - Southern Cone palm Syagrus romanzoffiana (Cham.) Glassman. The fate of the important palm collection gathered by Barbosa Rodrigues is here discussed and notes on the palms described in the publication are added in order to better define their taxonomic identity.
\end{abstract}

\begin{abstract}
Resumen
STAUFFER, F., L. RAMELLA, E. FERREIRA \& L. NOBLICK (2016). Lectotipificación de los nombres descritos por J. Barbosa Rodrigues en la obra "Palmae novae Paraguayenses". Candollea 71: 105-115. En español, resúmenes en inglés y español. DOI: http://dx.doi.org/10.15553/c2016v711a13

En el marco de la preparación de la familia Palmae a publicarse en la Flora del Paraguay, lectotipos son designados para los siguientes nombres publicados por J. Barbosa Rodrigues en la obra "Palmae novae Paraguayenses": Attalea guaranitica Barb. Rodr., Bactris anisitsii Barb. Rodr., Cocos paraguayensis Barb. Rodr. y Cocos sapida Barb. Rodr. Este último nombre es aquí propuesto como un nuevo sinónimo para la palma Syagrus romanzoffiana (Cham.) Glassman, ampliamente distribuida en el Sureste de Brasil y el Cono Sur. El destino de la importante colección de palmas acumulada por Barbosa Rodrigues es elucidado y se incluyen algunas notas que contribuyen a la mejor definición taxonómica de las especies descritas en esta obra.
\end{abstract}

\section{Keywords}

ARECACEAE - PALMAE - Barbosa Rodrigues - Paraguay

\footnotetext{
Direcciones de los autores :
}

FS, LR: Conservatoire et Jardin botaniques de la Ville de Genève, case postale 60, 1292 Chambésy, Suiza. E-mail: fred.stauffer@ville-ge.ch

EF: Núcleo de Pesquisas do Acre (INPA/NPAC), Estrada Dias Martins s/n, Parque Zoobotânico da UFAC, Bairro Distrito Industrial, CEP 69911-845 Rio Branco, Acre, Brasil. 


\section{Introducción}

E1 célebre botánico brasileño J. Barbosa Rodrigues (18421909) realizó una corta expedición a la región norte del río Paraguay entre los años 1898 y 1899. Durante su estadía en Paraguay coleccionó palmas en compañía de Juan Daniel Anisits (1856-1911), botánico de origen húngaro que residía en Paraguay desde hacía varios años. La colección de palmas que realizó Barbosa Rodrigues en el marco de su visita a Paraguay fue posteriormente enriquecida por un grupo de especímenes que le habría solicitado a Anisits para un estudio más detallado sobre las palmas de la región. Este conjunto de muestras constituyó el fundamento de la obra "Palmae novae Paraguayenses” publicada por Barbosa Rodrigues (1899).

De las 15 especies que Barbosa Rodrigues (1899) reporta como nativas de la flora paraguaya, siete de ellas fueron propuestas como nuevas para la ciencia. Considerando que la mayoría de estas especies muy bien pudieran estar presentes también en la región central de Brasil, el autor interpretó a esta publicación como un complemento de su obra "Palmae Mattogrossenses" (Barbosa Rodrigues, 1898).

El trabajo editorial que se adelanta para la publicación de la familia Palmae de la Flora del Paraguay puso en evidencia que una serie de aspectos taxonómicos y nomenclaturales asociados a la obra "Palmae novae Paraguayenses" debían ser previamente aclarados. La presente contribución tiene como objetivos:

1) Determinar el origen e identificar el destino de los especímenes sobre los cuales fueron basados los nombres propuestos en la obra,

2) Lectotipificar los nombres de la familia Arecaceae que fueron allí publicados siguiendo las recomendaciones de los artículos 9.11 y 9.12 del ICN Melbourne.

\section{Origen y destino de los especímenes citados en la obra "Palmae novae Paraguayenses"}

Como se ha explicado anteriormente, la obra "Palmae novae Paraguayenses" está basada en el conjunto de especímenes colectados por J. Barbosa Rodrigues y J.D. Anisits en el norte de Paraguay durante los años 1898-1899, así como del grupo de especímenes que Anisits le enviara posteriormente a Barbosa Rodrigues. Con el objeto de interpretar correctamente la identidad taxonómica de las especies descritas en la obra se hizo una investigación de los herbarios en los que al menos en teoría deberían encontrarse los tipos nomenclaturales asociados a estas especies: en nuestro caso principalmente en el herbario de palmas de Barbosa Rodrigues y alternativamente en algún herbario que hubiera podido conservar especímenes de Anisits. Algunas informaciones claves sobre el destino de cada una de estas colecciones se indican a continuación.
Las colecciones de palmas de Barbosa Rodrigues

El destino de las colecciones de palmas que Barbosa Rodrigues acumuló a lo largo de su prolífica carrera botánica es bastante confuso y las pocas informaciones disponibles son difícilmente verificables. Por razones que aun hoy en día se desconocen, aparentemente Barbosa Rodrigues prefirió conservar las muestras que acumulaba en su colección privada, en vez de depositarlas en algún herbario brasileño o extranjero ya establecido. El conflicto que lo habría opuesto durante largos años al botánico ingles James William Helenus Trail (18511919) podría estar vinculado, al menos parcialmente, a esta decisión (ver detalles en Mori \& Ferreira, 1987; SÁ, 2001); sin embargo, en ausencia de mayores evidencias es imposible determinar exactamente la motivación que lo llevó a no depositar su herbario en una institución publica.

La información asociada a los especímenes que Barbosa Rodrigues colectara en la región amazónica es igualmente bastante confusa. Sus exploraciones y colecciones en la región se iniciaron en 1872, año en el que bajo el patrocinio del Barón de Capanema (Guillerme Schuch de Capanema 1824-1909, hermano de Dom Pedro II), Barbosa Rodrigues es comisionado a explorar el valle del Río Amazonas (Mori \& Ferreira, 1987), teniendo entre otras obligaciones "completar, corrigir e aumentar o Gênero Palmarum do venerado Martius" (SÁ, 2001). Sin embargo, la consolidación de sus investigaciones amazónicas solamente fue evidente entre 1883 y 1889, periodo en el que fundó y ocupó el cargo de director del Museo Botánico de Amazonas y del Jardín Botánico de Manaus. Barbosa Rodrigues acumuló durante sus años amazónicos una importante colección de plantas (ca. 10000 especímenes), entre ellas muchas palmas, así como fósiles y artesanías indígenas. Aunque aparentemente varios cientos de documentos, entre libros y correspondencia, estarían aun conservados en la biblioteca del Instituto Nacional de Pesquisas da Amazônia (INPA), el destino de la importante colección de plantas acumuladas por Barbosa Rodrigues en esta región no ha podido ser elucidado, siendo el caso que Mori \& FERreira (1987) afirman que a falta de todo cuidado, la casi integralidad de las colecciones botánicas habría desaparecido una vez que cesaron las actividades del museo. No obstante, de acuerdo a Vatтimo-Gil (1979), algunas colecciones realizadas por Barbosa Rodrigues en su primer viaje al Amazonas han podido ser identificadas en el herbario del Jardín Botánico de Río de Janeiro (RB).

Mientras ocupaba el puesto de Director del Jardín Botánico de Rio de Janeiro, cargo al que fue nombrado en el año 1892, Barbosa Rodrigues conservó en su casa la mayor parte de la colección. Aun cuando Stafleu \& Cowan (1976) aseguran que la perdida de este herbario se debió a un incendio, nuevos indicios parecen sustentar que un número aún indefinido de las mismas habrían sido más bien destruidas en su casa de Rio de Janeiro durante una inundación. De igual 
manera, parecería que algunos especímenes sobrevivientes asociados a esta importante colección habrían, en ausencia de todo cuidado, desaparecido después la muerte de Barbosa Rodrigues (A. Peixoto, com. pers.).

En el marco de su prolífica carrera como experto de la familia Arecaceae Barbosa Rodrigues describió 4 géneros, 204 especies y 16 variedades como nuevos para la ciencia, en gran medida basados en el material que habría acumulado durante más de 30 años de estudios botánicos en Brasil y en menor grado en Paraguay. La pérdida de los tipos nomenclaturales sobre los cuales están basados todos estos nombres necesitará de un exhaustivo trabajo de tipificación (neotipificación o lectotipificación) similar al que presentamos en esta contribución.

\section{Las colecciones de palmas de J. D. Anisits}

Los especímenes de palmas de J. D. Anisits hacían parte de la colección privada de Barbosa Rodrigues, que como se ha indicado anteriormente fue destruida por razones aún poco definidas. De acuerdo a Kümmerle (1933) y Lanjouw \& STAFLEU (1954), las colecciones más importante de especímenes de Anisits parecen estar depositadas en los herbarios del Museo Húngaro de Historia Natural (BP) y del Museo Sueco de Historia Natural (S); sin embargo, especímenes aislados de este colector han sido también identificados en los herbarios B, F, G, y NY. De acuerdo a comunicaciones personales de Z. Barina (BP) y M. Ehn (S), ningún material de palmas asociado a Anisits pudo ser encontrado en estas colecciones, lo que apoya la hipótesis de que la integralidad de las colecciones de palmas que fueron enviadas a Barbosa Rodrigues, tuvieron el mismo destino que el resto de las colecciones de palmas del botánico brasileño.

\section{Lectotipificación de los nombres publicados por Barbosa Rodrigues en la obra "Palmae novae Paraguayenses"}

Cada especie incluida en el "Palmae novae Paraguayenses" presenta una extensa descripción morfológica, datos sobre su distribución geográfica y algunas notas sobre sus afinidades taxonómicas. En el caso de las nuevas especies el texto va igualmente acompañado por una ilustración realizada con gran destreza por el propio Barbosa Rodrigues y basada sobre material vivo, indicado en la ilustración: "Barb. Rodr. des. d'ap. nat.”. Todas estas ilustraciones presentan detalles importantes para la definición taxonómica de las especies y como indicado un poco más tarde algunas ya han sido empleadas para la lectotipificación de los nombres descritos en la obra. Por estas razones y para facilitar el acceso a estas ilustraciones que se encuentran poco difundidas, reproducimos aqui el conjunto de las tablas de "Palmae novae Paraguayenses" (fig. 1-6). Hemos conservado el orden de la tablas originales que corresponde a la numeración de las figuras del presente artículo.
En base a la casi certitud que el material original colectado por Anisits ha sido destruido junto al resto de la colección de Barbosa Rodrigues, una lectotipificación de los nombres incluidos en el "Palmae novae Paraguayenses" es indispensable para el trabajo que el "Conservatoire et Jardin botaniques de la Ville de Genève" viene realizando sobre la redacción de esta familia para la Flora del Paraguay. Las lectotipificaciones que se presentan a continuación son acompañadas por comentarios de importancia sobre la identidad taxonómica de los nombres. Se han incluido igualmente en negritas el nombre actualmente reconocido para cada taxón de acuerdo a Govaerts et al. (2016) y SoAres (2015) para el género Butia (Becc.) Becc., así como todas las recombinaciones homotípicas.

Attalea guaranitica Barb. Rodr., Palm. Paraguay. : 27. 1899 (fig. 4).

Lectotypus (in hoc loco designatus): tab. IV fig. D in Barb. Rodr., Palm. Paraguay. 1899 [Se corrige aquí la lectotipificación de Glassman (1977a: 44)].

= Attalea geraensis Barb. Rodr., P1. Jard. Rio de Janeiro 6: 22.1898 .

Notas. - Consideramos que la ilustración presentada en la publicación, conteniendo detalles de la bráctea peduncular y cortes longitudinales y transversales del fruto, debe ser retenida como lectótipo de la especie, siguiendo el artículo 9.12 del ICN Melbourne. Esto debe ser considerado como una corrección a la lectotipificación propuesta por GLASSMAN (1977a : 44) quien consideró al espécimen Hassler 1860 (G [G00009200]) como lectótipo de la especie. Es importante mencionar aquí que ninguna colección de E. Hassler sirvió de base para la publicación de la "Palmae novae Paraguayenses" y nuestra lectotipificación se basa en el material o ilustración originales directamente asociados a la descripción del nombre.

Bactris anisitsii Barb. Rodr., Palm. Paraguay.: 19.1899 (fig. 5).

Lectotypus (in hoc loco designatus): tab. V in Barb. Rodr., Palm. Paraguay. 1899 [Barbosa Rodrigues efectúa una transcripción errónea del nombre de Anisits ("anizitzii”) utilizando dos veces la letra "z" en lugar de "s" (LAJos, 1999: 13)].

= Bactris glaucescens Drude in Mart., Fl. Bras. 3(2): 345 . 1881.

Notas. - Bactris anisitsii fue propuesto como sinónimo de B. glaucescens Drude por Henderson (2000); sin embargo, tal como lo indicó en su revisión del género, este autor no estudió el material tipo asociado a la primera de estas especies. 


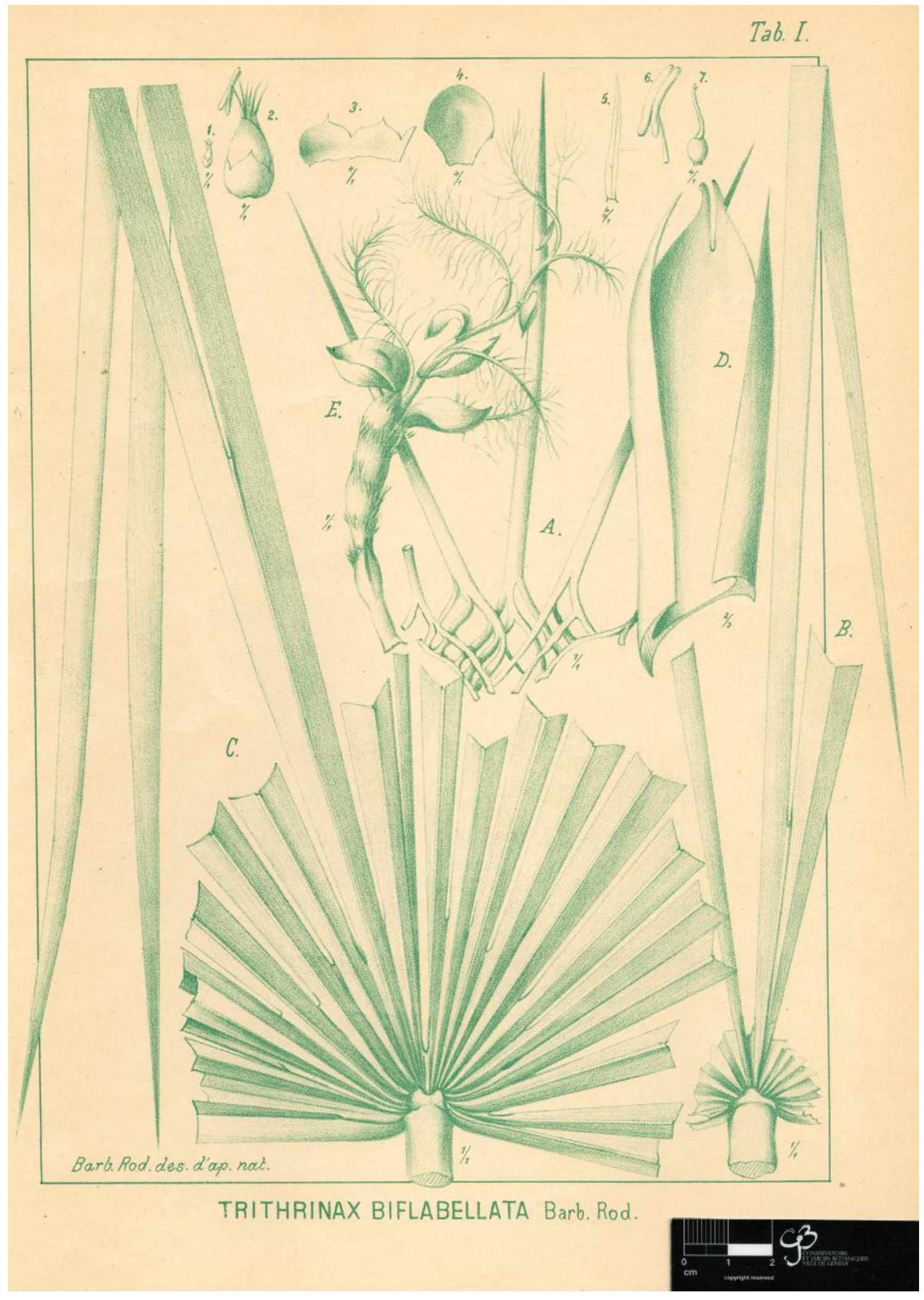

Fig. 1. - Lectotypus de Trithrinax biflabellata Barb. Rodr. [BARBOSA RODRIGUES, 1899: tab. I] 


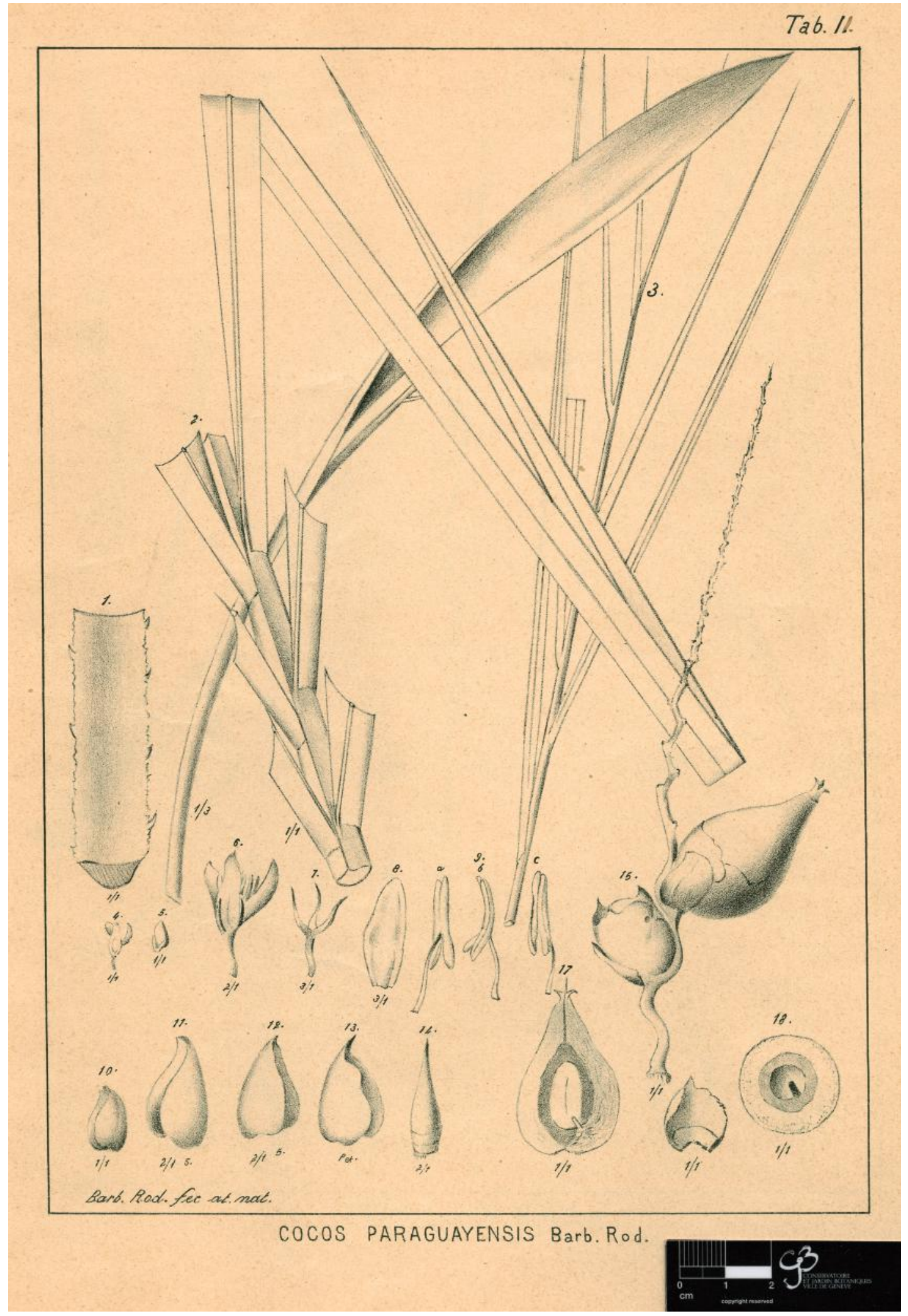

Fig. 2. - Lectotypus de Cocos paraguayensis Barb. Rodr.

[BARBOSA RODRIGUES, 1899: tab. II] 
Cocos paraguayensis Barb. Rodr., Palm. Paraguay.: 9. 1899 (fig. 2).

$\equiv$ Butia yatay var. paraguayensis (Barb. Rodr.) Becc. in Agric. Colon. 10: 503. 1916.

$\equiv$ Butia paraguayensis (Barb. Rodr.) L.H. Bailey in Gentes Herb. 4: 47. 1936.

$\equiv$ Syagrus paraguayensis (Barb. Rodr.) Glassman in Fieldiana, Bot. 32: 151. 1970.

$\equiv$ Butia yatay subsp. paraguayensis (Barb. Rodr.) Xifreda \& Sanso in Hickenia 2: 207. 1996.

Lectotypus (in hoc loco designatus) : tab. II in Barb. Rodr., Palm. Paraguay. 1899.

Notas. - En una etiqueta colocada por S. Glassman en 1968 se designa al ejemplar Hassler 896 (G [G00009205]) como lectótipo de C. paraguayensis; sin embargo, por las razones que se han explicado más arriba consideramos que la ilustración presentada en la "Palmae novae Paraguayenses" debe ser retenida como lectótipo de la especie. La interpretación taxonómica de esta palma sigue siendo muy compleja, en parte debido a evidentes incongruencias aparecidas en la obra "Sertum Palmarum Brasiliensum". En esta publicación, algunas dimensiones de órganos indicadas en la descripción de la especie (BARBosa Rodrigues, 1903 : 110) no corresponden con la ilustración (BArbosa Rodrigues, 1903: tab. 82). Las comparaciones que hemos realizado entre la descripción y la ilustración publicadas en el "Palmae novae Paraguayenses" demuestran por el contrario una gran coherencia, lo que de acuerdo a nuestro criterio representa una razón fundamental para considerar que la interpretación taxonómica de la especie debe basarse fundamentalmente en esta publicación.

Cocos sapida Barb. Rodr., Palm. Paraguay.: 12. 1899 (fig. 3), syn. nov.

Lectotypus (in hoc loco designatus): tab. III in Barb. Rodr., Palm. Paraguay. 1899.

= Syagrus romanzoffiana (Cham.) Glassman in Fieldiana, Bot. 31:382.1968.

Notas. - La identidad taxonómica de esta especie es aun en día bastante confusa, siendo el caso que la publicación de Govaerts et al. (2016) lo incluye dentro del grupo de nombres aun no atribuibles a un taxón conocido. El último autor de la presente contribución, Larry Noblick, realizó en el año 2004 una búsqueda infructuosa de esta especie en el Departamento de Misiones (Paraguay). La descripción y la ilustración de este nombre en "Palmae novae Paraguayenses" confirman su pertenencia al género Syagrus Mart. y manifiesta claras diferencias del resto de las especies de este género reportadas en la región. En la descripción original de la especie,
Barbosa Rodrigues indica que la palma tiene un porte de $2 \mathrm{~m}$ y una distribución que incluye los departamentos de San Salvador y Misiones. Estas informaciones pudieran ser atribuidas a la especie $S$. romanzoffana única especie de Syagrus que puede alcanzar esta altura y ocupa la región centro-sur de Paraguay. Esperando colectar informaciones adicionales sobre la identidad taxonómica de esta palma proponemos considerar a Cocos sapida como un sinonimo de Syagrus romanzoffiana. $\mathrm{El}$ hecho de que ninguna población de esta palma pudo ser descubierta por uno de los coautores pone en evidencia el elevado grado de amenaza al que están sometidas la mayoría de las palmas paraguayas (GAUTo et al., 2011).

Diplothemium anisitsii Barb. Rodr., Palm. Paraguay.: 16. 1899 (fig. 4).

$\equiv$ Allagoptera anisitsii (Barb. Rodr.) H.E. Moore in Principes $6: 38.1962$.

Lectotypus (designado por Moraes, 1996: 26) : tab. IV fig. A in Barb. Rodr., Palm. Paraguay. 1899 [Barbosa Rodrigues efectúa una transcripción errónea del nombre de Anisits ("anizitzii") utilizando dos veces la letra "z" en lugar de "s" (Lajos, 1999: 13)].

$=$ Allagoptera leucocalyx (Drude) Kuntze, Revis. Gen. P1. 2: 726.1891.

Scheelea quadrisperma Barb. Rodr., Palm. Paraguay.: 23. 1899 (fig. 6).

Lectotypus (designado por Glassman, 1977b : 240): tab. VI in Barb. Rodr., Palm. Paraguay. 1899.

= Attalea anisitsiana (Barb. Rodr.) Zona in Palms 46: 132. 2002.

Trithrinax biflabellata Barb. Rodr., Palm. Paraguay.: 2. 1899 (fig. 1).

$\equiv$ Trithrinax schizophylla var. biflabellata (Barb. Rodr.) An. Cano \& F.W. Stauffer in Phytotaxa 136: 42. 2013.

Lectotypus (designado por CANo et al., 2013: 42): tab. I in Barb. Rodr., Palm. Paraguay. 1899. 


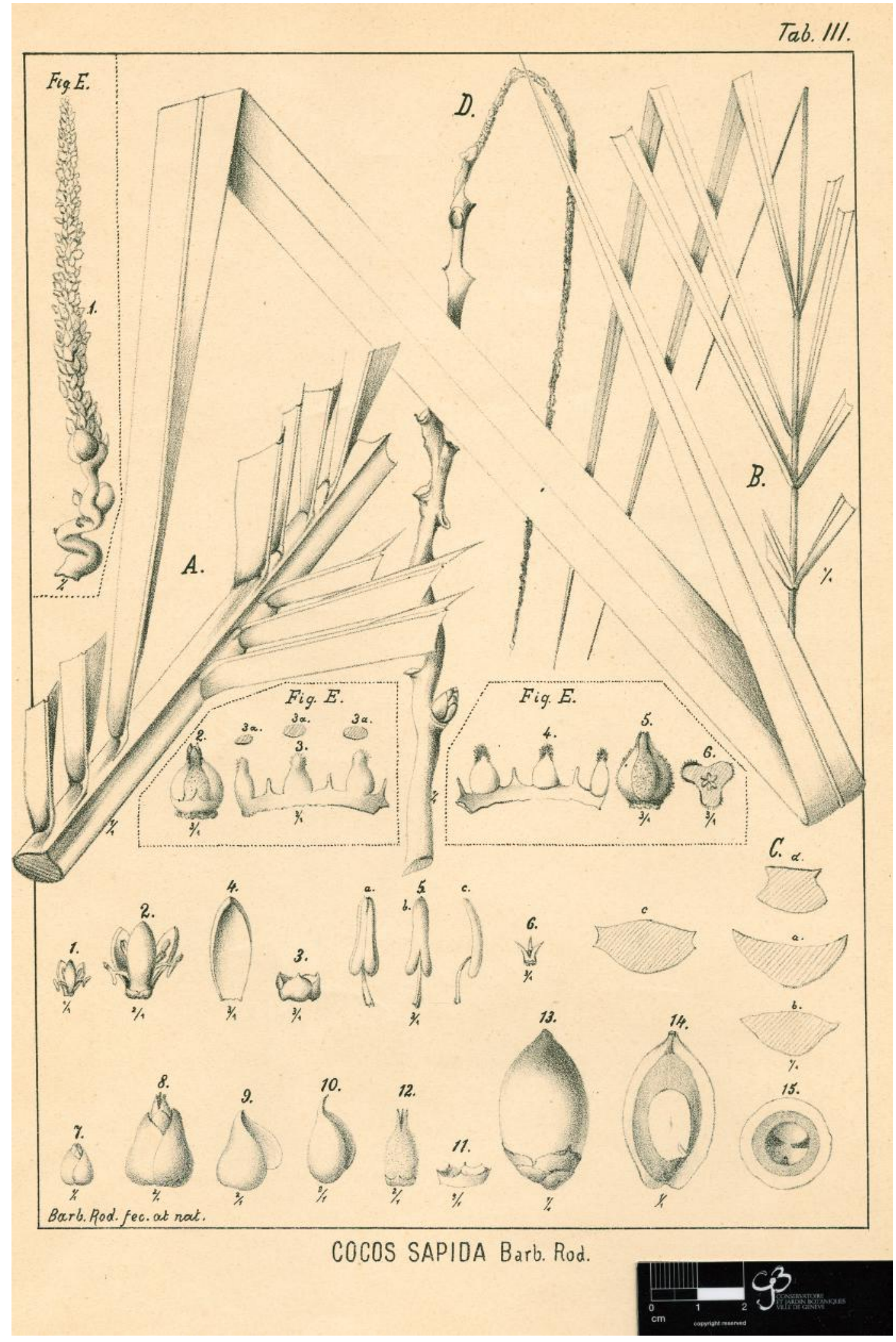

Fig. 3. - Lectotypus de Cocos sapida Barb. Rodr.

[BARBOSA RODRIGUES, 1899: tab. III] 


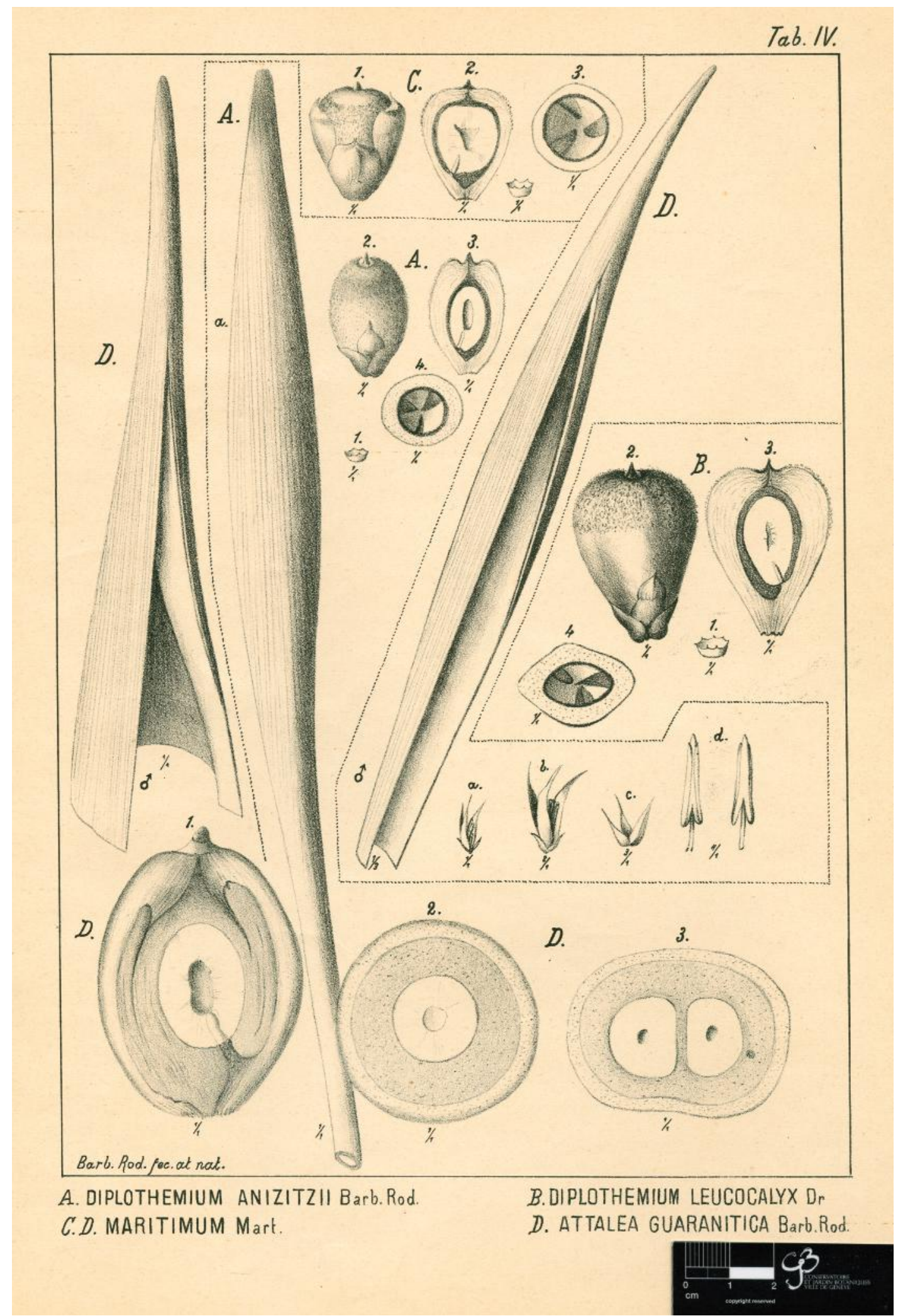

Fig. 4. - Lectotypus de Diplothemium anisitsii Barb. Rodr. (fig. A). Lectotypus de Attalea guaranitica Barb. Rodr. (fig. D). [BARBOSA RODRIGUES, 1899: tab. IV] 


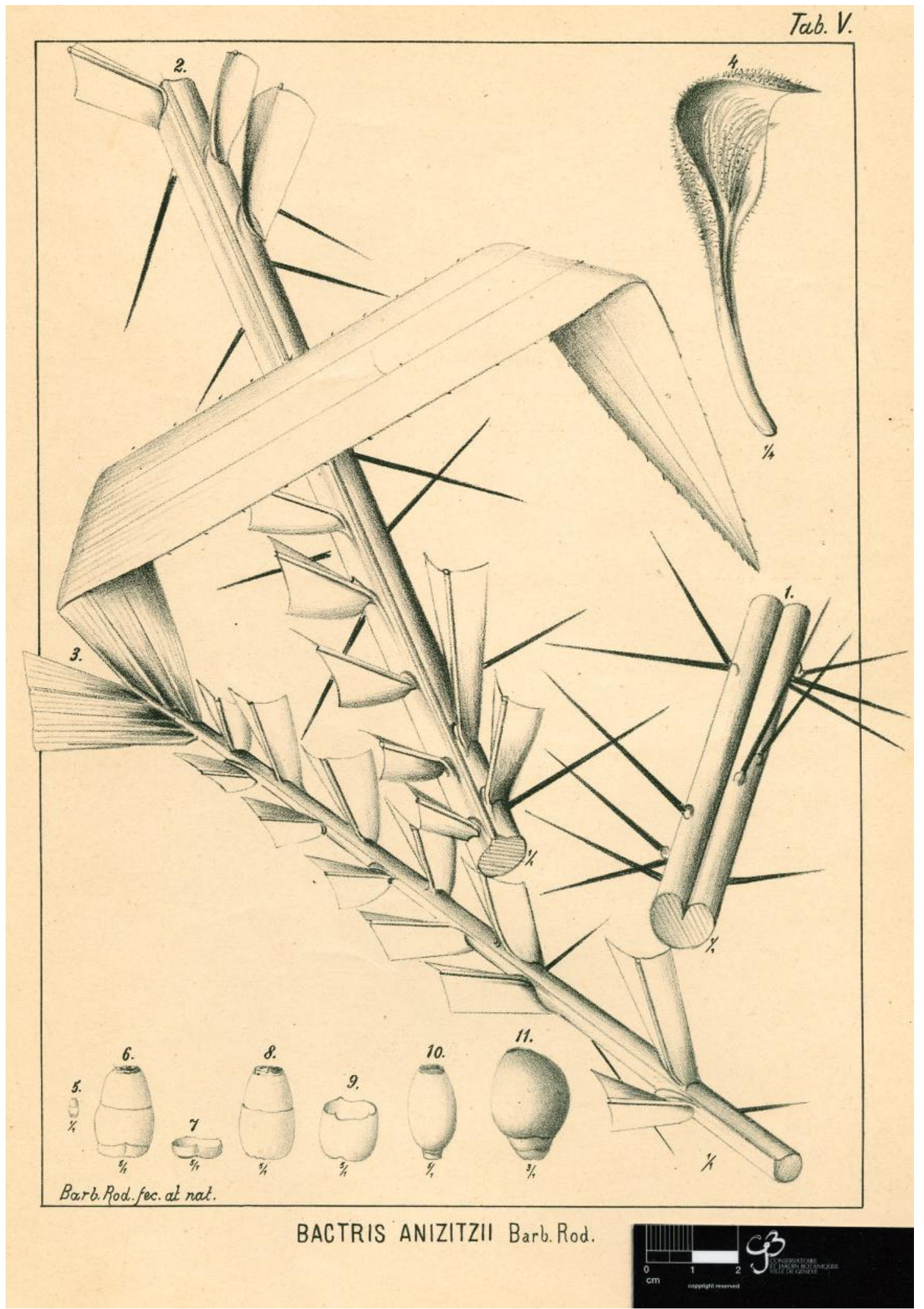

Fig. 5. - Lectotypus de Bactris anisitsii Barb. Rodr. 


\section{Agradecimientos}

Queremos agradecer la colaboración de Zoltán Barina (BP) y Mia Ehn (S) por la ayuda suministrada en cuanto a la búsqueda de muestras asociadas a J. D. Anisits. Ariane Peixoto (RJ) transmitió valiosa información sobre el destino de las colecciones de Barbosa Rodrigues en ese herbario.

\section{Referencias}

Barbosa Rodrigues, J. (1898). Palmae Mattogrossenses novae vel minus cognitae. Typographia Leuzinger, Rio de Janeiro.

Barbosa Rodrigues, J. (1899). Palmae novae Paraguayenses. Typographia Leuzinger, Rio de Janeiro.

Barbosa Rodrigues, J. (1903). Sertum Palmarum Brasiliensium. 2 vol. Veuve Monnom, Bruxelles.

Cano, A., M. Perret \& F.W. Stauffer (2013). A revision of the genus Trithrinax (Cryosophileae, Coryphoideae, Arecaceae). Phytotaxa 136: 1-53.

Gauto, I., R. Spichiger \& F.W. Stauffer (2011). Diversity, distribution and conservation status assessment of Paraguayan palms (Arecaceae). Biodivers. Conserv. 20: 2705-2728.

Glassman, S.F. (1977a). Preliminary taxonomic studies in the palm genus Attalea H.B.K. Fieldiana, Bot. 38: 31-61.

Glassman, S.F. (1977b). Preliminary taxonomic studies in the palm genus Scheelea Karsten. Phytologia 37 : 219-250.
Govaerts, R., J. Dransfield, S.F. Zona, D.R. Hodel \& A. HenDERSON (2016). World Checklist of Arecaceae. Royal Botanic Gardens, Kew [http://apps.kew.org/wcsp] [Consultado 15.1.2016].

Henderson, A. (2000). Bactris (Palmae). Fl. Neotrop. Monogr. 79.

Kümmerle, J.B. (1933). Die paraguayanischen PteridophytenSammlungen J. Daniel Anisits's. Magyar Bot. Lapok 32: 58-63.

Lajos, N. (1999). Anisits János Dániel emblékszám. To the János Dániel Anisits's memory, data for János Dániel Anisits's biography. Debreceni Pozsgástár num. spec.: 1-84.

LANJouw, J. \& F.A. StAFleu (1954). Index herbariorum: a guide to the location and contents of the world's public herbaria; part 2(1): collectors A-D. Regnum Veg. 2.

Moraes, M. (1996). Allagoptera (Palmae). Fl. Neotrop. Monogr. 73.

Mori, S.A. \& F.C. Ferreira (1987). A distinguished Brazilian botanist, João Barbosa Rodrigues (1842-1909). Brittonia 39 : 73-85.

SÁ, M.R. (2001). O botãnico e o mecenas : João Barbosa Rodrigues e a ciência no Brasil na segunda metade do século XIX. Hist. Ci. Saude Manguinhos 8: 899-924.

Soares, K. (2015). Le genre Butia. Princeps 1: 12-57.

Stafleu, F.A. \& R.S. Cowan (1976). Taxonomic litterature; volume I: A-G. Regnum Veg. 94: 119-120.

Vatтimo-Gil, I. (1979). Contribução au conhecimento da distribução geográfica das Lauraceae IV. Rodriguesia 49: 5-16. 


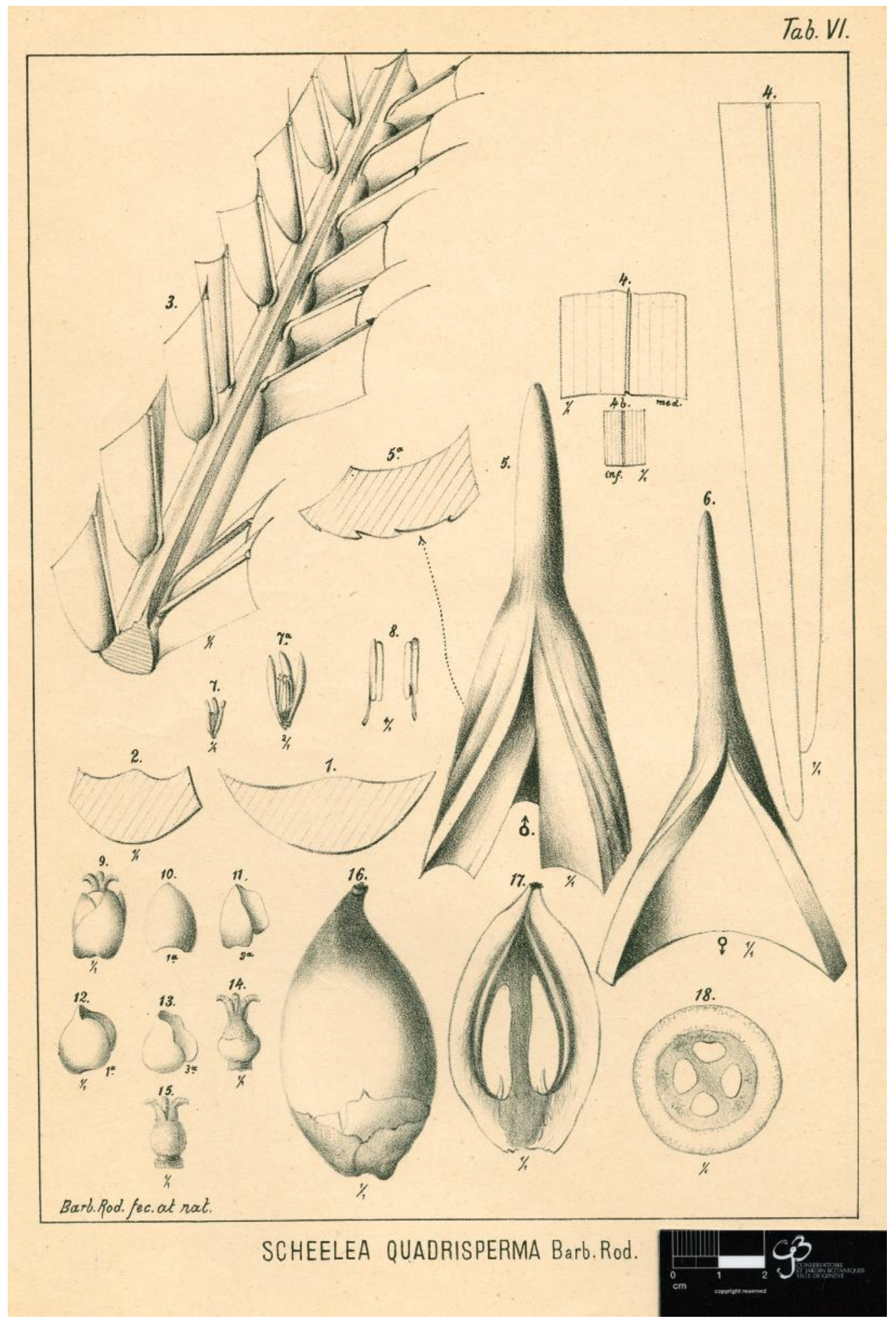

Fig. 6. - Lectotypus de Scheelea quadrisperma Barb. Rodr.

[BARBOSA RODRIGUES, 1899: tab. VI] 